To the Editor of Thl: British Journal of Ophthalmologi

SIR,-Owing to the courtesy of Mr. Tomlinson I have been allowed to see his criticism of my paper. I fully admit that the criticism is just in some special cases such as bank clerks, but I adhere to the opinion I expressed for those one usually meets with.

I find that I take two seconds to read a line of this journal, so I have to make every two seconds a to and fro lateral movement, i.e., a slow movement to the right followed by a rapid resilient, jerk to the left in order to start a new line. The length of these lines is about $11 \mathrm{~cm}$. , a distance occupied by 28 lines in height, so that the downward movement is in the first place unidirectional, and finally only $1 / 28$ of the extent of the lateral movement in the same time. This is the reason why I maintain that when reading it is important that the rapid lateral movement should be made by the eyes, and the vertical depression should, when necessary, be attained by depression of the head.

I am, however, very grateful to $\mathrm{Mr}$. Tomlinson for drawing attention to the special cases in which a rapid vertical movement is required.

Yours faithfully,

A. S. Percival.

\title{
CLASSIFICATION OF DISEASES OF THE CHOROID
}

To the Editor of The British. Journal of Ophthalmology

SIR,-In the September number of the Brit. Jl. of Ophthal., you publish a paper by Mr. Hepburn on the "Classification of Diseases of the Choroid," in which he specially attacks my attempts at classification of diseases of the macula, for which he says : "There is no justification," and accuses me of "confusion of thought."

Mr. Hepburn's paper was read before the Ophthalmological Section of the Royal Society of Medicine, and in the discussion which followed I made a reply, which, whatever its merits, showed that I neither accepted his criticism nor agreed with his conclusions. The publication of his paper without my reply might lead your wider field of readers to suppose that there was no other side to the question. I should be glad, therefore, if you would publish this letter. 
Mr: Hepburn claims that the classification of diseases of the fundus should be founded solely on a pathological basis, and I suppose he would extend this view to all other structures of the eye, and elsewhere-a high ideal, but not very helpful to the clinician.

As clinicians, our main object must be to find out the cause of the clinical manifestation that we observe, rather than to bring it under one of the pathological headings Mr. Hepburn suggests. Of course, it is desirable that we should recognize a "typical inflammatory scar" as such; but what is of far more importance is that we should be able to determine, either by position

- or other characters of the lesion, the probable cause of the inflammation.

I wonder at Mr. Hepburn making the statement that: "Fundus diseases ought to be classified according to the structure in which they occur, either retina or choroid, as both these structures are present in all parts of the fundus." If a mere clinician had made this statement, I should have expected him to be promptly called to order for his ignorance of the fact that, while the retina, for instance, covers the fundus, it differs in structure, vascular supply, and function, in its various zones; and the same may, I believe, be said of the choroid to a lesser extent.

Mr. Hepburn objects to the classification of lesions by their locality. But surely in this he is depriving us of a valuable aid to diagnosis of the cause of the disease. Not only in eye diseases, but in general medicine, the locality or structure picked out by certain toxins or drugs, is one of our important aids to diagnosis, and the symmetry or the one-sidedness of the lesion is often a determining factor. It is an absorbing question, this selection of certains cells or structures by different drugs, toxins, or microbes, for their manifestation, and one which I do not think even pathologists are prepared to answer.

Mr. Hepburn strikes me as ungrateful to the ophthalmoscopic observer, when he considers classification based on ophthalmoscopic examination as "a relic of the past," and it seems to me that the ophthalmoscopic observer can still-like the fool-ask more questions than the wise pathologist can answer.

In reply to $\mathrm{Mr}$. Hepburn at the meeting of the Section, in justifying my claim for a special place for the classification of disease of the macula as an entity, after summarizing some of the anatomical, physiological and pathological peculiarities of the macula, I limited myself to two points-demonstrating them by numerous ophthalmoscopic pictures-which, if conceded, would in my view establish my claim for a classification of macula diseases as such. 
These points were :

1. The extraordinary symmetry of true macula disease due to constitutional diseases, which appears to be almost a constant characteristic.

2. The establishment of cerebral macula disease as a definite entity.

If these two points be conceded, I fail to see how it can be held that other groups, such as septic, toxic and cardio-vascular, may not exist.

If it were a question of classifying the human race, $\mathrm{Mr}$. Hepburn would, presumably, do it by colour-black, white, yellow, etc. But would it show "confusion of thought" if another man did it by locality, speaking of Africans, Europeans, Chinese and Japanese, etc.? The colour classification may be the wider, but it does not deprive the geographical classification of its value. As a matter of fact, both are necessary. It is just as important to know the kind of man one may expect in China or Japan, as to recognize the characteristics of a yellow man when one meets him in the street. There is surely room, and need, for an anatomical, physiological basis of classification as well as a pathological, without showing any confusion of thought or casting "a distinct slur" on Mr. Hepburn and his fellow-pathologists.

Yours faithfully,

Rayner D. Batten.

LoNDON.

tonson.

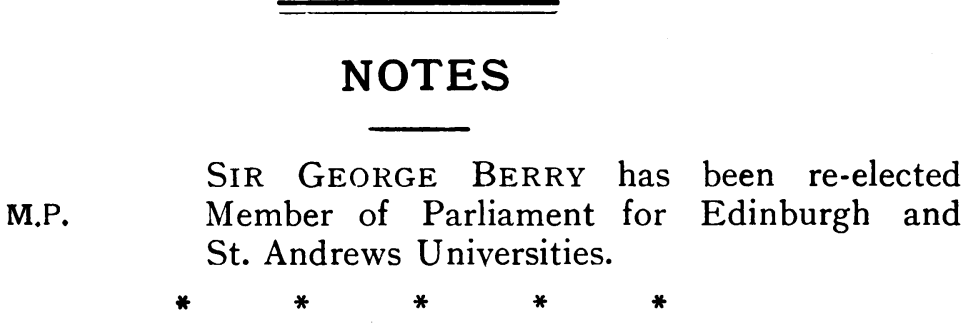

Course on Slit-Lamp

Microscopy of the Living Eye

A course upon the use of the slit-lamp is to be given at the Oxford Eye Hospital on December 19, 20, 22, and 23.

The course, which will be under the general direction of $\mathrm{Mr}$. P. Adams, Reader in Ophthalmology at Oxford University, will be modelled upon that given by Professor Vogt at Zürich.

The lectures will be given by Mr. Harrison Butler. Owing to the kindness of Messrs. Zeiss, eight slit-lamps will be at the disposal of the Hospital. Dr. Franceschetti, First Assistant to Professor Vogt, has accepted an invitation to act as Demonstrator. 